\title{
Low-Voltage Energy-Dispersive X-ray Spectroscopy and Electron Energy-Loss Spectroscopy Analysis of Presolar Graphite Spherules
}

Pierre Haenecour ${ }^{1}$, Jane Y. Howe ${ }^{1,2}$, Thomas J. Zega ${ }^{1,3}$, Sachiko Amari ${ }^{4}$, Christine Floss ${ }^{4}$, Paul Wallace ${ }^{1}$, Katharina Lodders ${ }^{5}$, Kazutoshi Kaji ${ }^{6}$, Takeshi Sunaoshi ${ }^{6}$, Atsushi Muto ${ }^{2}$.

1. Lunar and Planetary Laboratory (LPL), University of Arizona, Tucson, AZ, USA.

2. Hitachi High-Technologies America Inc., Clarksburg, USA.

3. Dept. of Materials Science and Engineering, University of Arizona, Tucson, AZ, USA.

${ }^{4}$ Physics Dept., and Laboratory for Space Sciences, Washington University, St. Louis, MO, USA.

5. Dept. of Earth and Planetary Sciences, Washington University, St. Louis, MO, USA.

6. Hitachi High-Technologies Corporation, Hitachinaka, Japan.

The application of transmission electron microscopy (TEM), energy-dispersive x-ray spectroscopy (EDS) and electron energy-loss spectroscopy (EELS) to planetary materials, such as meteorites, has allowed the investigation of the nature, microstructure and composition of their different constituents. In particular, combined TEM, EDS and/or EELS studies have provided unique insight into the composition, chemistry and mineralogy of extraterrestrial carbonaceous materials, such as presolar graphite grains [1-2]. However, C-rich materials can be sensitive to electron beam damage, e.g., organic matter analyzed at accelerating voltages above the displacement-threshold energy for $\mathrm{C}$ bonding [2-4]. The recent development of TEM/STEM instruments with lower accelerating voltages settings $(\leq 60 \mathrm{kV})$ allowed the mitigation of this problem. Moreover, the use of low accelerating voltages (e.g., 1-30kV) for STEM and EDS analysis also provides additional information on possible microstructural and compositional variations within the thickness of the sample cross-section that is not available at higher voltages. Here we report on new low-voltage $(\leq 30 \mathrm{kV})$ STEM, EDS and EELS data of presolar graphite spherules.

We prepared electron-transparent cross sections of five presolar graphite spherules, via previously described focused-ion-beam techniques [5], using the dual-beam Helios NanoLab 660 FIB-SEM at LPL: one grain (LAP-149) identified in-situ in a thin-section of the LAP 031117 chondrite [6] and four acidresidue grains from the Murchison CM chondrite [7]. We carried out EDS and EELS analysis on all six samples using a Hitachi SU9000 30kV SEM/STEM equipped with an Oxford Instruments X-Max 100LE EDS detector and a Hitachi EELS system. All measurements were carried out with a 30kV accelerating voltage. We carried out additional EDS analyses of a presolar graphite spherule at four different accelerating voltages: $1 \mathrm{kV}, 5 \mathrm{kV}, 10 \mathrm{kV}$ and $30 \mathrm{kV}$. Finally, we also acquired an EDS maps at $30 \mathrm{kV}$ in the vacuum just above the FIB section to identify all the possible system peaks.

Grain LAP-149 is about $1 \mu \mathrm{m}$ in diameter with a croissant-like shape and has extremely ${ }^{13} \mathrm{C}-$ rich and ${ }^{15} \mathrm{~N}-$ poor consistent with an origin in in the ejecta of a low-mass CO nova [6]. Bright-field (BF) and annular dark field (ADF) images reveal the presence of a rim between the bottom of the grain, which was not exposed to the NanoSIMS Cs ion beam and the surrounding matrix. EDS mapping suggests that this rim is composed of a mixture of carbonaceous material and silicate (elemental composition consistent with ferromagnesian silicate; Fig. 1). EELS measurements show that the carbonaceous material is amorphous. Because grain LAP-149 was identified in a pristine meteorite that experienced only minimal secondary processing (e.g., heating and aqueous alteration), this rim likely reflects grain surface processing in the interstellar medium or in the solar nebular before accretion of the meteorite parent body [8].

BF and ADF images also show the presence of inclusions inside three (LAP-149 and KFC1b2-gr1-a and 
-b) of the five presolar graphite grains studied (Fig. 1). EDS analysis reveals different elemental compositions for these three inclusions. The one in LAP-149 has heterogeneous distributions of Si and O across the inclusion, suggesting that it might be composed of several grains, including both ferromagnesian silicate and oxide grains. The inclusion in KFC1b2-gr1-a appears to be composed of two distinct phases: a Si-rich phase containing significant amount of Zr and Os surrounded by a Ti- and Morich oxide (Fig. 1). Finally, the inclusion inside KFC1b2-gr1-b has a major element composition consistent with $\mathrm{TiC}$, with similar amounts of $\mathrm{Ti}$ and $\mathrm{C}$, but also has detectable amounts of lithophile and siderophile elements ( $\mathrm{Zr}$ and Mo).

While many different types of refractory inclusions have previously been reported inside of presolar SiC and graphite grains [2], to our knowledge, this is the first identification of a possible presolar silicate grain inside of a presolar graphite grain. The $\mathrm{C}, \mathrm{N}$ and $\mathrm{S}$ isotopic composition of grain LAP-149 are consistent with a CO nova origin [6] and, thus, the identification of an O-rich inclusion inside this grain suggests changing redox conditions during condensation of LAP-149 in the ejecta of a low-mass CO nova [8].

\section{References:}

[1] E Zinner in "Treatise on Geochemistry", ed. H.D.H.K. Turekian, (Elsevier, Oxford), p. 181-213.

[2] T K Croat et al., Elements 10 (2014), p. 441-446.

[3] E Groopman et al., Geochimica et Cosmochimica Acta 221 (2018), p. 219-236.

[4] N D Bassim et al., Journal of Microscopy 245 (2012), p. 288-301.

[5] T J Zega et al. Meteoritics \& Planetary Science 42 (2007), p. 1373.

[6] P Haenecour et al., The Astrophysical Journal 825 (2016), p. 1-9.

[7] S Amari et al., Geochimica et Cosmochimica Acta 133 (2014), p. 479-522.

[8] This work is supported by NASA Grants NNX15AD94G (NExSS EOS program) and NNX15AJ22G, and NSF Grant 1531243. The work was carried out at the Kuiper Core Imaging and Microscopy Facility, University of Arizona.
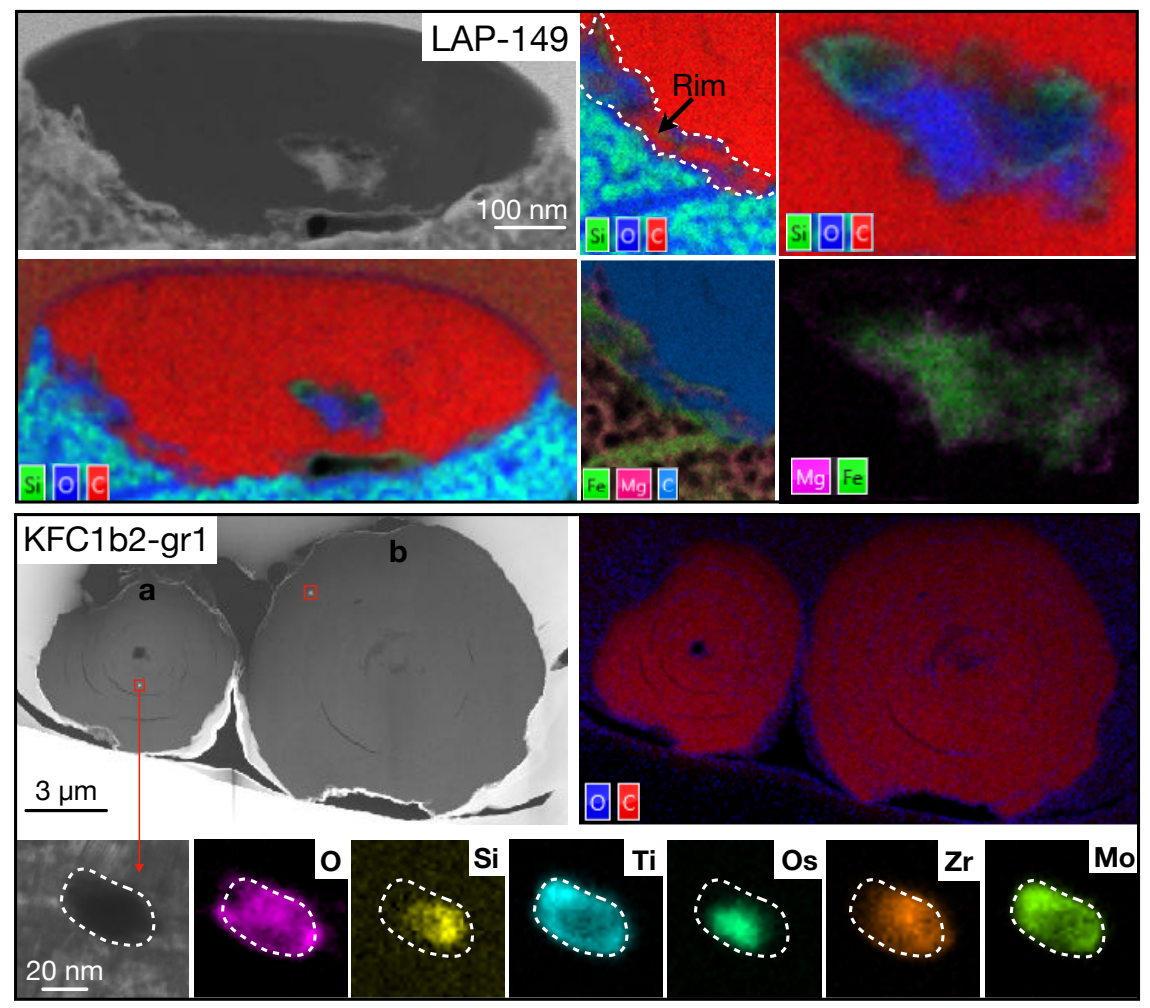

Fig. 1. ADF STEM images and falsecolor EDS elemental maps of graphite grains LAP-149 and KFC1b2-gr1-a and KFC1b2-gr1-b. 